\title{
Inteligência Artificial no Contexto da Indústria 4.0
}

\author{
Eduardo Santos Telles, Dante Augusto Couto Barone, Alexandre Moraes da Silva \\ Instituto de Informática - Universidade Federal do Rio Grande do Sul \\ Av. Bento Gonçalves, 9500 - Porto Alegre - RS, 91509-900 - Brasil \\ eduardo.telles@ufrgs.br, baronedinf.ufrgs.br, \\ alexandre.silva@inf.ufrgs.br
}

\begin{abstract}
With the migration of artificial intelligence (AI) from fiction to a disruptive technology applicable to production processes, there is a need to analyze how this theme is related to the fourth industrial revolution, known as industry 4.0. This paper aims to expose the aspects of each theme and relate them to generate the debate and demonstrate the existing applications.
\end{abstract}

Resumo. Com a migração da inteligência artificial (IA) da ficção para uma tecnologia disruptiva aplicável nos processos produtivos, existe uma necessidade de analisar como este tema está relacionado com a quarta revolução industrial, conhecida como indústria 4.0. Este trabalho tem como objetivo expor os aspectos de cada tema e relacioná-los para gerar o debate e demonstrar as possiveis aplicações existentes.

\section{Introdução à Inteligência Artificial Industrial}

A inteligência artificial (IA) é uma ciência cognitiva com pesquisas nas áreas de processamento de imagens, robótica, processamento linguagem natural, aprendizado de máquina etc. $\mathrm{O}$ desenvolvimento de novas tecnologias pode ser separado em fases, e no caso da IA, existem estudos que demonstram que uma nova onda de interesse por IA iniciou em meados de 2010 baseada em três fatores: I) Utilização de Big data para comércio eletrônico, redes sociais, pesquisas online e aplicações governamentais; II) Métodos de aprendizado de máquina e algoritmos que evoluem conforme o crescimento das bases de dados e III) Computadores potentes que fornecem o processamento necessário. Este progresso disruptivo está mudando a IA tradicional (IA 1.0), que pressupõem métodos simbólicos caracterizado por conteúdos estruturados e controle centralizado, para uma nova fase, chamada IA 2.0. A ênfase neste caso, é para o aprendizado de máquina, em especial deep learning, com conteúdo não estruturados, descentralizados (distribuídos aleatoriamente) e controle de estruturas (Yao et al. 2017).

A IA e aprendizado de máquina tem sido consideradas técnicas avançadas no setor industrial, porém existe falta de evidências que comprovem que estas técnicas funcionam e resultam em um retorno sobre o investimento atrativo para os acionistas das empresas. Ainda, os resultados dos algoritmos de aprendizado de máquina dependem da experiência dos desenvolvedores. Assim, o sucesso da aplicação da IA no setor industrial ainda é limitado, com barreiras e limites para desenvolver novas pesquisas que tornem este assunto uma disciplina com foco na criação, validação e uso de aprendizado de máquina para aumentar a eficiência produtiva. O desenvolvimento de pesquisas promove soluções para aplicações industriais e funciona como uma ponte que 
conecta pesquisas acadêmicas e industrias que aplicam IA (Lee et al. 2018; Zhong et al. 2017).

É esperado um crescimento da utilização de automação industrial baseada em IA, com resultados quantitativos positivos no índice de produtividade das empresas. Atualmente as indústrias enfrentam desafios em termos de demanda e competividade global, com constante necessidade por mudanças e utilização de novas técnicas produtivas. Um promissor conceito tecnológico é a nova revolução industrial, conhecido amplamente como Indústria 4.0 (termo oriundo da Alemanha) mas também difundido como Smart manufacturing (EUA) e Smart factory (Coreia do Sul) (Wuest et al. 2016). A integração da IA com emergentes pesquisas realizadas nas áreas de Internet of Things (IOT) (Rüßmann et al. 2015), Big data (Yao et al. 2017), Computação na nuvem (AlmadaLobo 2016) e Espaços cyber físicos (Wang 2016) possibilita que as operações industriais sejam mais flexíveis e eficientes.

A interação entre humanos e máquinas na indústria possibilita o trabalho colaborativo, com o uso de tecnologias cognitivas de aprendizado de máquina. Assim, avançados modelos de aprendizado de máquina são aplicados em robôs inteligentes, capazes de aprender com humanos e desenvolver aptidões complementares em diferentes contextos operacionais (Zhong et al. 2017). Estudos realizados pela Festo Bionic Learning Network encontrou muitas aplicações, como braços robóticos que utilizam IA para otimização do algoritmo e BionicANT, com utilização sistemas multiagentes para habilitar a comunicação de robôs entre si e encontrar a melhor maneira de organizar, de forma colaborativa, determinada tarefa proposta (Festo 2020).

Ao considerar que a IA na indústria 4.0 está em um processo inicial de crescimento é importante conhecer melhor sobre o assunto e suas aplicações. Para atingir este propósito, este trabalho tem como objetivo analisar um sistema produtivo que possua elementos da indústria 4.0 e forneça melhor compreensão sobre as relações da IA neste ambiente. A contribuição deste trabalho é gerar o debate e demonstrar as possíveis aplicações existentes, de modo que pesquisadores e pessoas relacionadas à indústria possam explorar as aplicações possíveis.

\section{Elementos da Inteligência Artificial Industrial}

Os elementos da IA Industrial incluem: I) Tecnologia Analítica; II) Tecnologia de Big Data; III) Tecnologia de Computação na nuvem; IV) Domínio do Conhecimento e V) Evidência (Lee et al. 2018). A tecnologia analítica é um elemento essencial da IA, que por si só não contribui se os demais elementos não existirem. Big Data e Tecnologia de Computação na nuvem são essenciais para fornecerem dados para o sistema de IA. Já Domínio de Conhecimento e Evidência são elementos que possibilitam compreender o problema, fornecer dados relevantes para o sistema de IA, preparar o sistema para coletar corretamente e com qualidade os dados, conhecer as conexões existentes do sistema e os parâmetros de produção exigidos e, além de tudo, validar os dados gerados pelo sistema de IA.

\section{Benefícios da Inteligência Artificial Industrial}

A aplicação dos elementos de IA em conjunto possibilita que os sistemas de manufatura aprendam com as próprias experiências, com propósito de permitir a solução autônoma de problemas e otimizar a produção (Zhong et al. 2017). A coleta de dados do sistema 
produtivo e posterior identificação de padrões, possibilita que a IA Industrial crie modelos cada vez mais precisos e robustos com o tempo. A figura 1 (adaptado de Lee et al. 2018) demonstra a comparação da eficiência entre um sistema industrial tradicional e com IA ao longo do tempo.

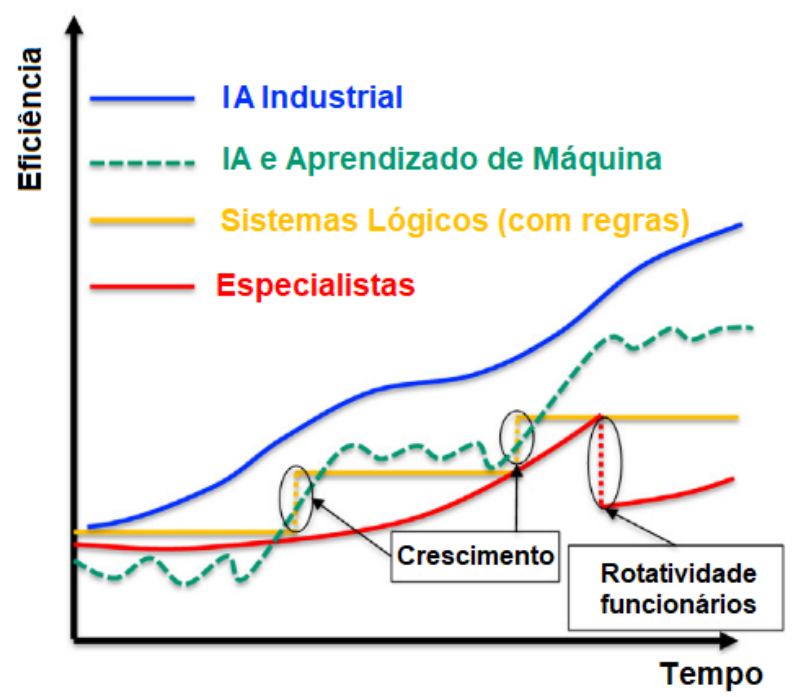

Figura 1. Comparação da IA Industrial com outros métodos de aprendizado

O crescimento da eficiência baseada em Especialistas apresenta o menor ganho para as indústrias, uma vez que após superar a curva de aprendizado em determinado momento irá ocorrer a rotatividade do funcionário e, por consequência, perda de parte do conhecimento adquirido previamente. Em relação aos Sistemas Lógicos, considera que o crescimento existe apenas em momentos que novas regras e parâmetros de ajustes são inseridos nos sistemas que suportam o incremento da eficiência. Já os sistemas que possuem IA, com capacidade de resolver problemas, apresentam crescimento após identificar oportunidades de otimização em relação ao cenário atual.

A aplicação de IA Industrial possibilita uma configuração dinâmica do arranjo de produção, não limitada a uma linha de produção fixa convencional com produtos idênticos, mas uma linha de produção modular que se ajusta conforme a combinação dos pedidos de produção recebidos dos clientes. A organização da linha de produção ocorre virtualmente nos Espaços cyber físicos. A aplicação de IA Industrial em fábricas da BMW nos processos de estampagem, pintura e montagem demonstraram aumento da eficiência, com redução de $30 \%$ no uso de água, redução de $40 \%$ no uso de energia e redução de 20\% nas emissões para atmosfera (Cheng et al. 2017).

\section{O Sistema Produtivo na Indústria 4.0}

Um modelo genérico sobre a integração dos diferentes elementos da indústria 4.0 é proposto por Zhong et al. (2017), no qual a integração dos processos é essencial. Tipicamente adotam-se sensores avançados, comunicação sem fio, processamento de grandes dados e algoritmos específicos para prover a troca de dados. Para implementar estas ferramentas, os sistemas produtivos buscam plataformas virtuais para simular e produzir os diferentes produtos customizados sem aumento de custo. Tais plataformas podem reduzir os custos ao otimizar a infraestrutura existente, com novas configurações de desenvolvimento, produção e gerenciamento logístico. 
A figura 2 (adaptado de Zhong et al. 2017) apresenta as relações existentes na indústria 4.0, sendo elas:

I) Design inteligente: Busca o rápido desenvolvimento de produtos com auxílio de novas tecnologias, como realidade virtual (VR - Virtual Reality) e realidade aumentada (AR - Augmented Reality). Softwares de desenvolvimento CAD (Computer-aided design) e CAM (Computer-aided manufacturing) possuem funções específicas para interagir com os Espaços cyber físicos em tempo real.

II) Máquinas inteligentes: A utilização de máquinas inteligentes pode ser alcançada com o auxílio de robôs inteligentes e vários outros objetos capazes de coletar informações e comunicar-se uns aos outros em tempo real. Por exemplo, uma máquina inteligente capaz de coletar dados e enviá-los para um sistema de computação na nuvem, para quando próximo do término de processamento de uma peça sincronize com as operações posteriores.

III) Controle em tempo real: Um sistema de produção adaptativo pode ser alcançado com a utilização de espaços cyber físicos. Controles inteligentes executam comandos para otimizar os resultados em diferentes máquinas inteligentes através de um sistema de computação na nuvem.

IV)Cronograma inteligente: A programação da produção pode ser otimizada incluindo avançados modelos e algoritmos com dados coletados dos sensores e máquinas. A capacidade de cálculo, modelagem e simulação de cenários possibilita melhor utilização dos recursos.

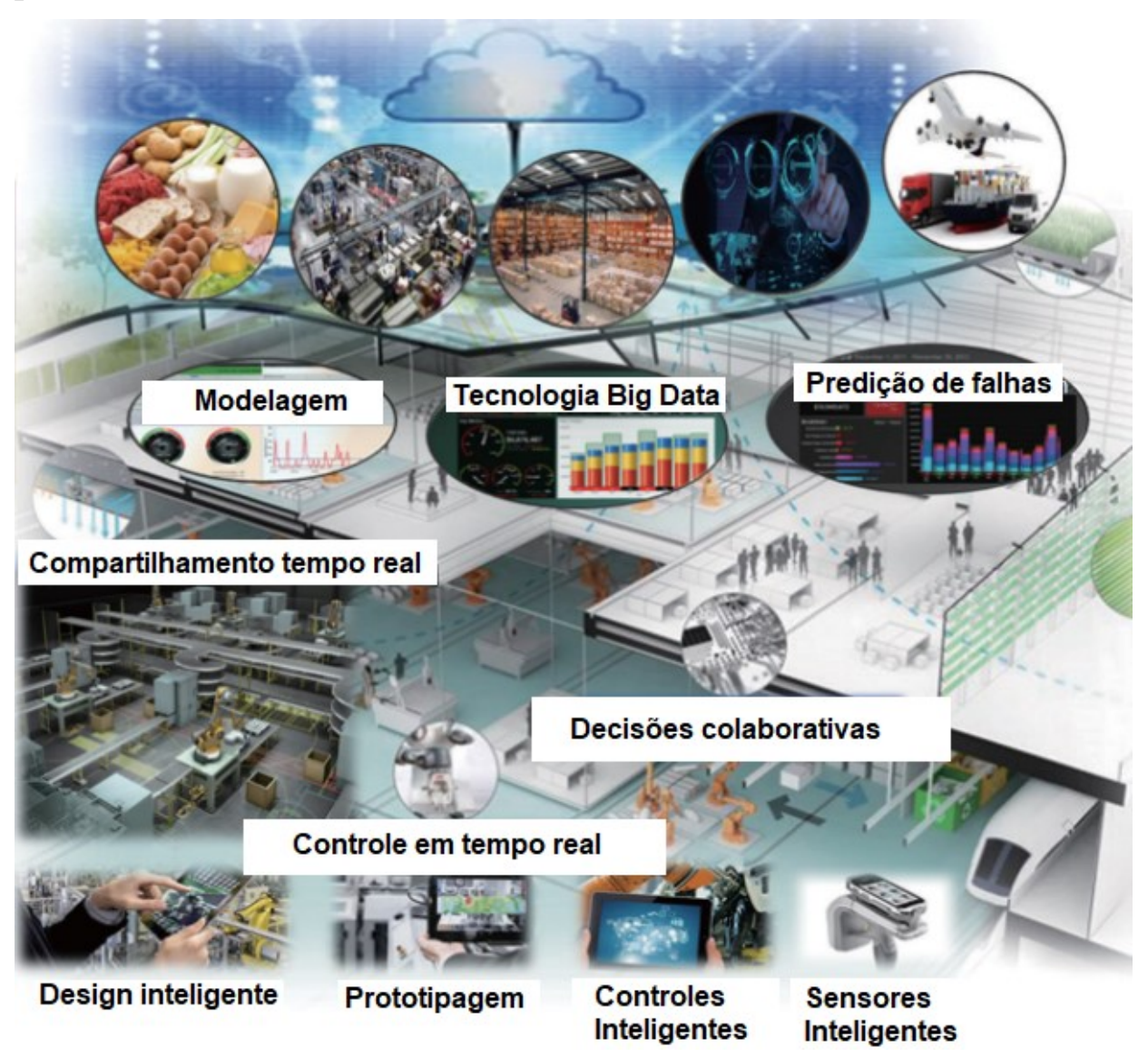

Figura 2. Indústria 4.0 


\section{Desafios da Inteligência Artificial Industrial}

A indústria é um setor de transformação estabelecido nas maiores economias mundiais, no entanto, países desenvolvidos identificaram uma redução da contribuição deste setor no resultado do produto interno bruno (PIB) nas últimas décadas. A concorrência global e redução nos custos de produção impõem novos desafios ao sistemas produtivos atuais (Wuest et al. 2016).

Os novos sensores, dispositivos eletrônicos e diferentes máquinas digitais utilizadas nos processos demandam grande capacidade de armazenamento de dados, em que frequentemente são perdidos ou não aproveitados plenamente. Executivos de empresas pioneiras nos setores em que atuam estão utilizando a tecnologia de Big data para otimizar as operações e prover decisões em tempo real (Zhong et al. 2016). A multinacional General Eletric (GE) utiliza a tecnologia de Big data para melhorar a comunicação com seus clientes e operações produtivas, no centro de monitoramento localizado em Atlanta. Um time com mais de 50 engenheiros analisam os dados que chegam de turbinas e geradores localizados em 58 países (Minter 2015).

A comunicação dos dispositivos e transmissão de dados exige que as estruturas atuais suportem as novas aplicações desenvolvidas. Os métodos de transmissão de dados via rede interna pode apresentar instabilidade, em especial quando utilizadas redes sem fio para transferir grande quantidade de dados. Sinais via rádio frequência e infravermelho são facilmente afetados por interferências eletromagnéticas, reflexão em metais e sobreposição de sinais durante a comunicação. A transmissão de dados depende da largura de banda e o protocolo utilizado, o atual modelo de comunicação móvel (ex. internet $4 \mathrm{G}$ ) não pode oferecer conexões eficientes e confiáveis para a tecnologia Big data (Zhong et al. 2016).

Os desafios, oportunidades e perspectivas futuras sobre IA Industrial foram classificados pelo autor em:

I) Tecnologia Big data: A capacidade de coletar dados, classificá-los, armazená-los e interpretá-los com o uso de IA pode se tornar a vantagem competitiva das indústrias. Para viabilizar a utilização desta tecnologia, as empresas precisam investir em redes de computadores potentes, métodos de proteção de dados, capacidade de armazenamento prevendo o crescimento e aumento da velocidade de processamento com novos computadores.

II) Modelagem de cenários: Os sistemas de IA que interpretarem com maior confiabilidade das informações serão capazes de auxiliar as empresas no aumento da eficiência. A modelagem de cenários pode ser realizada nos espaços cyber físicos, com elementos autônomos e cooperativos que interagem entre si com habilidade de fazer previsões para otimizar as máquinas e o sistema logístico das indústrias. A fusão entre os sistemas reais e virtuais possibilitam um sistema produtivo inteligente robusto em meio as mudanças e incertezas do ambiente. 
III)Predição de falhas: A identificação e predição de falhas em sistemas dinâmicos é uma extensão das informações coletadas pela tecnologia Big data. Novas técnicas de IA podem auxiliar com o aprendizado de máquina, baseado em dados coletados e histórico de falhas dos equipamentos. Esta área de estudo possibilitará aumento da disponibilidade e, por consequência, melhor aproveitamentos dos recursos fabris.

Aumentar a aplicação de IA industrial é um desafio que requer ferramentas e métodos confiáveis. Wuest (2016) comenta que outro desafio é levar em consideração a interpretação dos resultados. A maioria dos dados de saída não possui formas ilustradas de fácil compreensão, mas sim algoritmos e parâmetros de ajustes específicos com informações valiosas. Sem a correta interpretação dos resultados o impacto negativo pode ser prejudicial para o sistema produtivo. A capacidade analítica e julgamento de informações dos profissionais que atuam com IA são indispensáveis para prover os gestores dos processos industriais de informações concisas.

\section{Conclusão}

Com o aumento da discussão sobre os temas inteligência artificial e indústria 4.0, a análise dos elementos e iterações existentes é cada vez mais importante para compreender os novos sistemas produtivos que serão praticados. A indústria 4.0 é considerado um caminho sem volta, no qual exige adaptação das empresas que buscam continuar competitivas no mercado globalizado. A aplicação de técnicas de IA nos processos produtivos possibilita maior otimização dos resultados, com predição dos potenciais modos de falhas, aprendizado contínuo e, assim, aumento da eficiência.

Espera-se que este trabalho inspire novos pesquisadores e empresários de indústria em busca do conhecimento constante e contribua com o avanço dos processos produtivos. Também se acredita que os aspectos discutidos neste trabalho despertem novas ideias e gere discussões colaborativas para a aplicação das técnicas da indústria 4.0 nas empresas.

\section{Referências}

Almada-Lobo, Francisco. 2016. "The Industry 4.0 Revolution and the Future of Manufacturing Execution Systems (MES)." Journal of Innovation Management 3(4): 16-21.

Cheng, Guo Jian, Li Ting Liu, Xin Jian Qiang, and Ye Liu. 2017. "Industry 4.0 Development and Application of Intelligent Manufacturing." Proceedings - 2016 International Conference on Information System and Artificial Intelligence, ISAI 2016: 407-10.

Festo. 2020. "Bionic Learning Network." https://www.festo.com/group/en/cms/10156.htm (March 29, 2020).

Lee, Jay, Hossein Davari, Jaskaran Singh, and Vibhor Pandhare. 2018. "Industrial Artificial Intelligence for Industry 4.0-Based Manufacturing Systems." Manufacturing Letters 18: 20-23.

Minter, Steve. 2015. "GE Plans for Big Data Are 'Brilliant."” Industry week. 
https://www.industryweek.com/technology-and-iiot/emergingtechnologies/article/22005985/ge-plans-for-big-data-are-brilliant (March 27, 2020).

Rüßmann, Michael et al. 2015. "Industry 4.0. The Future of Productivity and Growth in Manufacturing." Boston Consulting (April): 1-5.

Wang, Ben. 2016. "The Evolution and Future of Manufacturing : A Review." Journal of Manufacturing Systems 39(April): 79-100.

Wuest, Thorsten, Daniel Weimer, Christopher Irgens, and Klaus Dieter Thoben. 2016. "Machine Learning in Manufacturing: Advantages, Challenges, and Applications." Production and Manufacturing Research 4(1): 23-45.

Yao, Xifan, Jiajun Zhou, Jiangming Zhang, and Claudio R. Boer. 2017. "From Intelligent Manufacturing to Smart Manufacturing for Industry 4.0 Driven by Next Generation Artificial Intelligence and Further On." Proceedings - 2017 5th International Conference on Enterprise Systems: Industrial Digitalization by Enterprise Systems, ES 2017: 311-18.

Zhong, Ray Y., Stephen T. Newman, George Q. Huang, and Shulin Lan. 2016. "Big Data for Supply Chain Management in the Service and Manufacturing Sectors: Challenges, Opportunities, and Future Perspectives." Computers and Industrial Engineering 101: 572-91. http://dx.doi.org/10.1016/j.cie.2016.07.013.

Zhong, Ray Y., Xun Xu, Eberhard Klotz, and Stephen T. Newman. 2017. "Intelligent Manufacturing in the Context of Industry 4.0: A Review." Engineering 3(5): 61630. http://dx.doi.org/10.1016/J.ENG.2017.05.015. 